\title{
CAMBIOS Y CONTINUIDADES EN LA ESCUELA SECUNDARIA: ANÁLISIS DE PROGRAMAS RECIENTES EN EUROPA Y AMÉRICA LATINA DESDE UNA PERSPECTIVA HISTÓRICA E INTERNACIONAL
}

\author{
Changes and continuities at secondary school: analysis of recent programs in \\ Europe and Latin America from historical and international perspective.
}

\section{Felicitas Acosta}

Fecha recepción: 21/09/2016

Fecha aceptación: 06/11/2016

RESUMEN: El objetivo de este artículo es analizar programas de cambio para la escuela secundaria en algunos países de Europa y América Latina. Propone para ello considerar las características de la expansión de la escuela secundaria en algunos países de los espacios territoriales antes mencionados con especial énfasis en la configuración histórica de modelos institucionales de dicha escuela. El argumento principal toma como eje la corroboración de la existencia de problemas comunes en los sistemas educativos de Occidente en relación con el par expansión-modelo institucional. En la presentación de los programas analizados, se señalan avances y límites en las tendencias de cambio mientras que en las conclusiones se sostiene la necesidad de analizar dichas tendencias desde una perspectiva histórica, sistémica e internacional. Esta perspectiva contribuye a considerar los efectos que las formas históricas de organización de la escuela secundaria tuvieron y tienen sobre las posibilidades y las formas de cambio en dicha escuela, entre ellas el refuerzo de la segmentación educativa y la estigmatización escolar.

PALABRAS CLAVE: Enseñanza secundaria, política de la educación, estudio transnacional

ABSTRACT: The overall aim of this article is to analyse recent educational programs towards secondary education in some countries of Europe and Latin America. For this purpose, it studies the characteristics of secondary school expansion in the regions afore mentioned. The main argument revolves around the existence of problems common to Western educational systems specifically in relation to the expansion of secondary schools and their institutional model. The presentation of the cases under analysis identifies advances and limits regarding the possibility of change in secondary schools organization. In this sense, conclusions point out the need to adopt a historical, systemic and international perspective in order to consider the effects of the historical organization of secondary school on the possibilities and characteristics of actual change, chief among them the reinforcement of educational segmentation and school stigmatization.

KEY WORDS: Secondary education, educational policy, transnational study 


\section{Introducción}

Las políticas de expansión de la escolarización secundaria históricamente han encontrado problemas de ajuste entre los modelos institucionales y los nuevos sectores sociales que acceden a ese nivel educativo; problemas que se manifiestan en el abandono escolar. Frente a ello, los países de modernización educativa temprana adoptaron diferentes estrategias para resolverlos en tanto la mayoría de los países de la región latinoamericana todavía enfrentan los problemas más básicos de la extensión de la secundaria (infraestructura, finalización de niveles educativos previos, formación de docentes entre otros).

Más allá de estas diferencias, propias del desarrollo histórico de los sistemas educativos, estudios previos indican que hay una forma histórica de organización de la escuela secundaria que se encuentra en la base de dichos problemas (véase Acosta, 2011a; Acosta, 2011b; Acosta, 2013; Acosta y Terigi, 2015). Esta forma histórica también estaría asociada a las reformas educativas que tendieron a la incorporación de más jóvenes en la secundaria desde los años '60 del siglo XX hasta la actualidad, tanto en los países de Europa Occidental como en países de América Latina (véase, entre otros, Weiler, 1998; Viñao, 2002; Wiborg, 2009). Algunos países avanzaron más que otros en atender este problema. Sin embargo, todos los países, más allá de su grado de avance, presentan casos de políticas educativas orientados hacia esta problemática.

El objetivo de este artículo es analizar programas de cambio para la escuela secundaria en algunos países de Europa y América Latina. Propone para ello considerar las características de la expansión de la escuela secundaria en algunos países de los espacios territoriales antes mencionados con especial énfasis en la configuración histórica de modelos institucionales de dicha escuela. Interesa analizar los posibles efectos de las formas de organización histórica de la escuela secundaria sobre los programas dirigidos hacia el cambio en la actualidad.

Para ello, el artículo se organiza en tres apartados que siguen a esta introducción. El primero presenta un enfoque conceptual desde una perspectiva histórica e internacional para el análisis de programas de cambio en la escuela secundaria. El segundo ofrece una sistematización de los principales programas estudiados, así como una reflexión sobre sus principales aportes. En el tercer apartado se analizan los alcances y los límites de las estrategias bajo análisis al momento de considerar la posibilidad del cambio en la escuela secundaria.

\section{El estudio de la expansión de la escuela secundaria en clave histórica e internacional}

Desde mediados del siglo XX la expansión de la escuela secundaria se instaló en la agenda de la política educativa de los estados nación; principalmente de aquellos que habían logrado universalizar la enseñanza primaria desde comienzos de dicho siglo. En este apartado se propone como argumento la existencia de problemas comunes en los sistemas educativos de Occidente en relación con el par masificación-organización de la escuela secundaria: ciertas características de su organización estarían asociadas a la dificultad para asegurar trayectorias exitosas para todos los estudiantes. Fueron las políticas (las reformas) las que dieron cuenta del desajuste entre la obligatoriedad (forzar la expansión) y la forma de organización (Acosta, 2011a y 2013).

De acuerdo con esta hipótesis, a medida que el completamiento de niveles básicos de escolarización fue presionando sobre la expansión de la educación secundaria, ciertas características de la organización tradicional de ésta generaron dificultades para retener a 
todos los estudiantes y asegurar la certificación de los estudios. De esta manera, las políticas de extensión de la escolarización secundaria han encontrado problemas de ajuste entre los modelos institucionales y los nuevos sectores sociales que acceden a ese nivel o lo que aquí se denomina el problema de la expansión frente al modelo institucional de la escuela secundaria.

La reformulación del problema del cambio en la escuela secundaria en términos del par expansión-modelo institucional parte de un marco histórico comparado con énfasis en tres dimensiones:

- La dimensión histórica: herencias institucionales que se arrastran entre modelos y prácticas y que dan forma a las instituciones educativas (Acosta, 2014; Tröhler y Lenz, 2015).

- La dimensión sistémica: dinámica sobre la que se estructuran las prácticas de escolarización, específicamente los procesos de sistematización y segmentación (Mueller, 1992; Ringer, 1992; Viñao, 2002; Acosta, 2011b y 2014).

- La dimensión ligada a los procesos de internacionalización de la educación: esto es la relación entre tendencias globales y prácticas nacionales en la expansión de la escolarización (Schriewer 2002 y 2010).

Esto quiere decir que para comprender el problema del cambio en la escuela secundaria es necesario considerar estas tres dimensiones. La construcción de un marco de este tipo se apoya en algunos supuestos. Se parte de la tesis del proceso de internacionalización de ideas y modelos en la configuración de los sistemas educativos en general y de la escuela secundaria en particular. El concepto de internacionalización, tal como lo desarrolla Schriewer $(2002 ; 2010)$, refiere al proceso de migración, de difusión y de recepción transnacional de discursos y prácticas educativas, proceso constituido históricamente en una variedad de lógicas de apropiación determinadas por estructuras culturales profundas (Caruso y Tenorth, 2011). Supone, al mismo tiempo, la difusión global de guiones educativos estandarizados transnacionalmente y la persistencia de varias redes de interrelación sociocultural (Schriewer, 2010).

Respecto de la configuración de los sistemas educativos y la escuela secundaria, se toman como puntos de partida los siguientes procesos de internacionalización:

- El triunfo de una forma escolar a fines de siglo XIX bajo las variables de la estatalización, la simultaneidad, la gradualidad, la constitución de nuevos oficios (docentes y alumnos).

- La articulación de instituciones educativas bajo procesos de sistematización y segmentación como matriz de configuración de los sistemas educativos (Mueller, 1992; Ringer, 1992).

- La configuración de la escuela secundaria como institución con rol diferencial en el proceso de segmentación educativa a partir dos elementos: su propio modelo institucional y su expansión a partir de modelos institucionales con funciones de instituciones determinantes (Steedman, 1992).

En este punto cabe precisar dos procesos sobre los que se configuraron los sistemas educativos que pueden ofrecer elementos para evaluar la forma en que se produjo la extensión de la enseñanza secundaria. A mediados de los años '80 Mueller (1992) desarrolló los conceptos de sistematización y segmentación para caracterizar los orígenes y la dinámica de los sistemas educativos. La sistematización refiere al proceso que tuvo lugar desde fines del siglo XIX de articulación entre instituciones educativas dispersas a través de la conjunción de los planes de estudio, los exámenes y las certificaciones. La segmentación, por su parte, 
indica que a medida que las instituciones se articulaban entre sí formando los niveles educativos, aquellas dirigidas a la enseñanza secundaria se diversificaban.

La escuela secundaria participó históricamente del proceso de segmentación a través de dos vías. Por un lado, un modelo institucional selectivo producto de herencias institucionales y procesos de impresión de modelos sobre otras escuelas. En efecto, las escuelas secundarias son herederas de un modelo -el de los colegios humanistas- del que reciben contenidos y finalidades -la enseñanza de las humanidades para las élites-; es decir conllevan un rasgo selectivo (Acosta, 2011b). En el momento en que comienza la lenta expansión de la enseñanza secundaria, esta tiende a realizarse sobre la base de las escuelas más cercanas al modelo antes descrito por su fuerte valoración social. De esta manera, y como destaca Viñao (2002), todas las escuelas secundarias siguieron una tendencia generalista que excluyó ciertos saberes y que tendió a concentrarse en la oferta de "Bachillerato".

Por otro lado, el direccionamiento de las trayectorias de los estudiantes a través de recorridos educativos ligados al lugar a ocupar en el mundo del trabajo. La investigación educativa luego mostró que ese lugar estaba en asociación con la posición social de origen. La segmentación actuó por diferencias inter institucionales (entre tipos y modalidades) e intrainstitucionales (ramas paralelas, secciones, turnos, clases avanzadas entre otras).

Los sistemas educativos de modernización temprana, como los de los países de Europa Occidental y algunos del Cono Sur (Argentina y Uruguay, por ejemplo) se configuraron bajo la dinámica arriba descripta. Se trata de sistemas de configuración sobre la base de la sanción de leyes de obligatoriedad escolar para el nivel primario y la creación de circuitos escolares, de acuerdo con el lugar a ocupar en el mundo del trabajo, para el nivel secundario. Durante la pos-guerra comenzó la expansión fuerte de este nivel educativo en buena parte de los países de Europa Occidental (véase al respecto Fernandez Enguita, 1985; Weiler, 1998; Campbell \& Sherrington, 2006; Acosta \& Pinkasz, 2007; Wiborg, 2009). América Latina siguió un ritmo diferente en este sentido, con avances en la expansión y dificultades en la graduación (Terigi, 2012; Acosta, 2013; Acosta y Terigi, 2015).

En ambos casos, las políticas de extensión de la escolarización secundaria han encontrado problemas de ajuste entre los modelos institucionales y los nuevos sectores sociales que acceden a ese nivel; problemas que suelen traducirse en el aumento de las tasas de repitencia y desgranamiento y en el descenso de las tasas de graduación y en el abandono temprano.

De acuerdo con un estudio de Wilson, Tanner-Smith, Lipsey, Steinka-Fry y Morrison (2011), en los países desarrollados las tasas de finalización de la escuela secundaria superior varían entre el $90 \%$ en Israel al $49 \%$ en Islandia. En los Estados Unidos el porcentaje de jóvenes de una misma cohorte que no se gradúa en se ubica entre el 13 y el $44 \%$ de acuerdo con el Estado; la media es de $26.8 \%$ con una tasa de finalización del $88 \%$. En Canadá la proporción de jóvenes entre 20 y 24 años que no completaron la secundaria y no están en la escuela es del 9\%. Países de Europa también muestran variaciones: Holanda y Francia presentan una tasa de finalización entre 80 y $85 \%$; los países nórdicos se ubican entre el $78 \%$ (Finlandia) y el 65\% (Dinamarca); Italia tiene la tasa más baja para el conjunto de países relevados en ese estudio con un $64 \%$.

Por su parte, la comisión Tackling early school leaving in Europe de la Unión Europea calcula que en Europa uno de cada 7 estudiantes abandona la escuela tempranamente y destaca el objetivo de reducir a un $10 \%$ la cantidad de jóvenes que abandonan la escuela sin completar sus estudios (Estrategia 2020; para 2011 se calculaba en 13.5\% la tasa de abandono temprano) y aumentar a un 40\% el logro de estudios superiores (European Commission, 2012 y 2014; Lyche, 2010). 
Respecto de América Latina, la asistencia a educación secundaria fluctúa enormemente al interior de los países según el nivel socioeconómico: desde un 93,6\% entre los más ricos hasta un 78,9\% entre los más pobres, en promedio para la región. Circa 2006, solo el 48,5\% de los jóvenes de 20 años había culminado el nivel medio (SITEAL, 2010), con situaciones críticas donde los porcentajes descienden hasta el 19,8\% (Honduras) o al 26,4\% (Nicaragua): entre los 17 y 18 años (edad de finalización teórica del nivel secundario en la mayoría de los países de América Latina), cerca de la mitad de los jóvenes ya no acuden a la escuela. En coherencia con lo señalado respecto a la limitada capacidad de cambio de la estructura que dio origen a la escuela secundaria, se detectan todaíva los procesos de selección interna que se encuentran en su matriz fundacional: a partir de los 13 años de edad comienza a observarse claramente el incremento sostenido de la proporción de adolescentes que abandona la escuela (SITEAL, 2010).

En síntesis, los estudios realizados sobre la escuela secundaria y la experiencia de las reformas educativas en las últimas décadas indican que el análisis de la expansión de la escuela secundaria debe contemplar, al mismo tiempo, las configuraciones históricas particulares, la heterogeneidad de los escenarios aún al interior de un mismo país o región y los problemas específicos de la masificación de la escuela secundaria, entre los que la revisión del modelo institucional ocupa un lugar central. Los programas que se presentan y analizan en el apartado siguiente apuntan hacia esa dirección.

\section{Programas recientes de cambio para la escuela secundaria: características y alcances}

Tal como se propuso en el apartado anterior, la educación secundaria presenta una dificultad histórica: su origen selectivo y excluyente frente a procesos de expansión. Algunos países avanzaron más que otros en atender este problema. Sin embargo, todos los países, más allá de su grado de avance, presentan casos de políticas educativas orientados a atender esta problemática.

En la actualidad, las transformaciones en las economías nacionales y regionales ubican a la educación secundaria como un factor crítico para acceder a la fuerza de trabajo. El abandono escolar antes de completar los estudios secundarios disminuye las posibilidades de integración al mundo del trabajo y aumenta las posibilidades de desempleo o de obtener salarios más bajos a lo largo de la vida. Por ejemplo, el National Dropout Prevention Center/Network (citado en Wilson, et al, 2011) de los Estados Unidos destaca que quienes abandonan la escuela secundaria ganan en promedio U\$S 9.245 menos que sus pares que sí terminan, tiene tasas de desempleo superiores en casi 13 puntos, se encuentran más representados en las poblaciones de las cárceles y son más proclives a ser padres adolescentes y vivir en la pobreza.

Existen muchos programas que prestan atención al problema del abandono escolar. La revisión de la literatura distingue tres estrategias principales: programas de re-estructuración de la escuela o el aula, programas focalizados en la formación y orientación hacia el trabajo y programas académicos suplementarios (Acosta, 2011a; Acosta, 2013; Acosta y Terigi, 2015; Wilson et al., 2011; Lyche 2012). Respecto del primero, de presencia creciente en países como los Estados Unidos, incluye distintas variantes, pero el supuesto principal es que la creación de una enseñanza más personalizada y relevante generará mayor implicación por parte de los alumnos. La variante más simple es la reducción del tamaño del grupo clase o la relación profesor/alumno. La más compleja supone una combinación de las anteriores con espacios de enseñanza personalizada, cursadas en bloque, las denominadas academias centradas en un grado y los programas de tipo schools-within-schools (edificios más 
pequeños, grupos de trabajo de 10 estudiantes liderados por guías o referentes de aprendizaje).

La estrategia vinculada con la orientación hacia el trabajo también apunta a crear experiencias educativas más relevantes para los estudiantes. Incluye programas tales como la formación vocacional, los cursos orientados hacia el trabajo, la orientación vocacional, pasantías y empleos pagos. Este tipo de estrategia es de particular relevancia en Europa.

En el caso los programas académicos suplementarios, comunes en los Estados Unidos y en Europa, constituyen una estrategia orientada hacia el abandono escolar. Sus formas más características son las tutorías, la educación remedial, la asistencia en la tarea escolar.

Muchas de estas estrategias se desarrollan en combinación con programas de desarrollo social los que demandan una articulación entre diferentes instituciones: comunitarias, de formación terciaria, del mundo del trabajo. Los programas para padres adolescentes ocupan un lugar cada vez más importante dadas las dificultades que supone mantener la escolarización para jóvenes que se encuentran en esa situación. En los Estados Unidos, por ejemplo, se encuentra una fuerte asociación entre el embarazo adolescente y el abandono escolar de las mujeres principalmente (Wilson et al., 2011).

El estudio de Wilson et al antes mencionado revela que ninguna categoría de programa sea significativamente más efectiva que la otra, aunque se destaca que los programas centrados en el monitoreo o control de asistencia escolar, si bien dan resultados positivos, son menos exitosos que aquellos orientados hacia la re-estructuración de la escuela, desarrollo de habilidades o formación para estudios superiores. La calidad de la implementación del programa sí aparece como un factor de relevancia, de allí la recomendación de seleccionar estrategias que puedan implementarse en las escuelas.

\subsection{Selección de programas}

A partir de los estudios relevados más arriba, se detectaron 4 tipos de programas respecto de los problemas más persistentes en la escolarización de adolescentes y jóvenes desarrollados en los últimos 10 años:

- Programas destinados al sostenimiento de la escolarización de los jóvenes centradas sobre el apoyo para el aprendizaje

- Programas destinados al sostenimiento de la escolarización de los jóvenes centradas sobre las condiciones sociales de los estudiantes

- Programas destinados a la finalización de la escuela secundaria

- Programas destinados a los cambios en la organización y la enseñanza en la escuela secundaria

En función de estos tipos de programas, para este trabajo se consideraron 4 criterios al momento de seleccionar los programas a analizar:

- La escala del programa implementado diferenciando entre políticas de tipo intensivas y políticas de tipo extensivas (Terigi, 2012).

- La atención a aspectos problemáticos pero diferentes de la escolarización de adolescentes y jóvenes: se incluyen programas de re ingreso a la escuela secundaria y programas de acompañamiento y sostenimiento (prevención del abandono) de la escolarización. En este sentido todos los programas deben ofrecer certificación escolar.

- La focalización sobre el problema del modelo institucional de la escuela secundaria: se incluyen aquellos programas que con mayor o menor grado de alcance consideran 
aspectos del modelo institucional (organizacionales y/o académicos) como parte de la estrategia de cambio.

- La evaluación del programa seleccionado: se trabaja con programas que cuentan con procesos de evaluación interna y/o externa con el fin de ofrecer ejemplos cuyos avances y dificultades se sustenten sobre información empírica. alfabético):

A partir de los criterios señalados se seleccionaron los siguientes programas (en orden

- Apoyo a Liceos (Chile).

- Centro de escolarización secundaria para adolescentes y jóvenes (CESAJ, Provincia de Buenos Aires, Argentina).

- Combatiendo el abandono escolar [Aanval op de uitval] (Holanda).

- Planes de Mejora Institucional (PMI, Argentina).

- Programa Aulas Comunitarias (PAC, Uruguay).

- Programa de impulso a la universalización (PIU, Uruguay).

- Programa Èxit (Barcelona, España).

- Programa para la finalización de los estudios [School Completion Programme] (SCP, Irlanda).

Los programas seleccionados se clasificaron en dos categorías de interés para el marco conceptual de este trabajo: programas de "sostenimiento de la trayectoria escolar" y programas de "re-ingreso a la escuela secundaria". En el primer caso, se optó por el concepto de "sostenimiento de la trayectoria escolar" en lugar del de prevención del fracaso en tanto se considera, como se señaló en el primer apartado, que el problema radica en el modelo institucional y en los arreglos institucionales necesarios para que la escuela "sostenga" y no expulse a sus alumnos. Se incluyeron en esta categoría los programas destinados a acompañar la escolaridad a través de dispositivos de acercamiento entre el alumno y la organización escolar.

La segunda categoría refiere a los programas implementados para promover la reinserción de los jóvenes al sistema formal y a la escuela secundaria por medio del desarrollo de espacios alternativos que se articulan con instituciones ya existentes o la creación de un nuevo tipo de oferta institucional.

A continuación, se presenta una tabla comparativa con las principales características de los programas seleccionados.

Tabla 1. Sistematización de principales características de programas seleccionados

\begin{tabular}{|c|c|c|c|c|c|c|c|c|}
\hline \multirow{2}{*}{$\begin{array}{l}\text { Tipo } \\
\text { Caso }\end{array}$} & \multicolumn{4}{|c|}{ Sostenimiento de la trayectoria escolar } & \multicolumn{4}{|c|}{ Re-ingreso a la escuela secundaria } \\
\hline & $\begin{array}{c}\text { Período de } \\
\text { implement } \\
\text { ación }\end{array}$ & $\begin{array}{l}\text { Población } \\
\text { objeto }\end{array}$ & $\begin{array}{l}\text { Ejes de la } \\
\text { intervención }\end{array}$ & $\begin{array}{l}\text { Líneas de } \\
\text { acción }\end{array}$ & $\begin{array}{c}\text { Período de } \\
\text { implementación }\end{array}$ & Población objeto & $\begin{array}{c}\text { Ejes de la } \\
\text { intervención }\end{array}$ & $\begin{array}{l}\text { Líneas de } \\
\text { acción }\end{array}$ \\
\hline 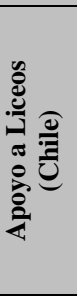 & $\begin{array}{l}2006 \quad y \\
\text { cont. }\end{array}$ & $\begin{array}{l}\text { Escuelas } \\
\text { alumnos } \\
\text { vulnerabili } \\
\text { zados y } \\
\text { bajos } \\
\text { resultados }\end{array}$ & $\begin{array}{l}\text { *Mejorar } \\
\text { resultados } \\
\text { en } 3 \text { años } \\
\text { *Mejorar } \\
\text { gestión y } \\
\text { capacidad } \\
\text { institucion } \\
\text { al }\end{array}$ & $\begin{array}{l}\text { *Programa } \\
\text { de asistencia } \\
\text { a demanda } \\
\text { de liceo en } \\
\text { el área } \\
\text { institucional } \\
\text {, pedagógica } \\
\text { y } \\
\text { convivencia }\end{array}$ & & & & \\
\hline
\end{tabular}




\begin{tabular}{|c|c|c|c|c|c|c|c|c|}
\hline \multirow{2}{*}{$\begin{array}{l}\text { Tipo } \\
\text { Caso } \\
\end{array}$} & \multicolumn{4}{|c|}{ Sostenimiento de la trayectoria escolar } & \multicolumn{4}{|c|}{ Re-ingreso a la escuela secundaria } \\
\hline & $\begin{array}{c}\begin{array}{c}\text { Período de } \\
\text { implement } \\
\text { ación }\end{array} \\
\end{array}$ & $\begin{array}{c}\begin{array}{c}\text { Población } \\
\text { objeto }\end{array} \\
\end{array}$ & $\begin{array}{c}\begin{array}{c}\text { Ejes de la } \\
\text { intervención }\end{array} \\
\end{array}$ & $\begin{array}{c}\text { Líneas de } \\
\text { acción }\end{array}$ & $\begin{array}{c}\begin{array}{c}\text { Período de } \\
\text { implementación }\end{array} \\
\end{array}$ & Población objeto & $\begin{array}{c}\text { Ejes de la } \\
\text { intervención }\end{array}$ & $\begin{array}{c}\text { Líneas de } \\
\text { acción } \\
\end{array}$ \\
\hline 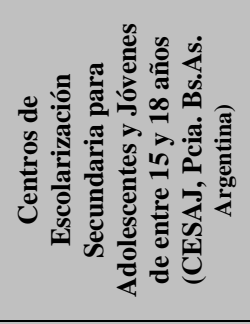 & & & & & 2008 y cont. & $\begin{array}{l}\text { Jóvenes } 15 \text { a } \\
18 \text { fuera del } \\
\text { SE (50 } \quad(50 \\
\text { CESAJ, } 1000 \\
\text { estudiantes a } \\
\text { 2011) }\end{array}$ & $\begin{array}{l}\text { *Re } \\
\text { ingreso en } \\
\text { ciclo } \\
\text { superior } \\
\text { *Aceleraci } \\
\text { ón de } \\
\text { trayectoria } \\
\text { s } \\
\text { *Vínculo } \\
\text { con trabajo }\end{array}$ & $\begin{array}{l}\text { *Espacio } \\
\text { puente } \\
\text { *Cambios } \\
\text { régimen } \\
\text { académico } \\
\text { *Diseño } \\
\text { curricular de } \\
\text { ciclo básico } \\
\text { específico } \\
\end{array}$ \\
\hline 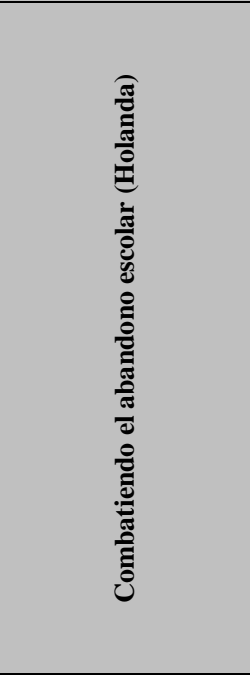 & $\begin{array}{l}2007 \text { y } \\
\text { continúa. }\end{array}$ & $\begin{array}{l}\text { Estudiantes } \\
\text { en la } \\
\text { educación } \\
\text { obligatoria } \\
\text { con } \\
\text { especial } \\
\text { atención a } \\
\text { la } \\
\text { transición } \\
\text { y } \\
\text { continuida } \\
\text { d pos } \\
\text { primaria. }\end{array}$ & $\begin{array}{l}\text { *Reducir } \\
\text { las tasas de } \\
\text { abandono } \\
\text { escolar. }\end{array}$ & $\begin{array}{l}\text { *Sistema de } \\
\text { seguimiento } \\
\text { de los } \\
\text { estudiantes } \\
\text { (número } \\
\text { personal). } \\
\text { *Portal del } \\
\text { digital de } \\
\text { absentismo. } \\
\text { *Coordinad } \\
\text { ores de } \\
\text { cuidado: } \\
\text { asistencia } \\
\text { socioeducati } \\
\text { va. } \\
\text { *Programas } \\
\text { de } \\
\text { continuidad } \\
\text { educativa, } \\
\text { orientación } \\
\text { laboral. }\end{array}$ & & & & \\
\hline 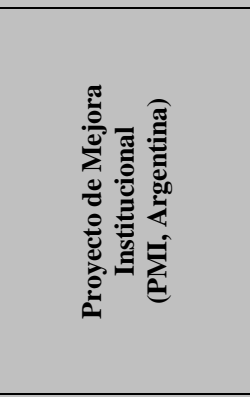 & $\begin{array}{l}2006 \text { y } \\
\text { cont. }\end{array}$ & \begin{tabular}{|l} 
Escuelas \\
secundaria \\
s (5254 en \\
$2011)$
\end{tabular} & $\begin{array}{l}\text { *Fortalecer } \\
\text { trayectoria } \\
\text { s escolares } \\
{ }^{*} \text { Generar } \\
\text { cambios en } \\
\text { la } \\
\text { propuesta } \\
\text { escolar y } \\
\text { organizaci } \\
\text { ón } \\
\text { institucion } \\
\text { al } \\
\end{array}$ & $\begin{array}{l}\text { *Espacios } \\
\text { extra clase } \\
\text { *Tutorías } \\
\text { *Articulació } \\
\text { n niveles } \\
\text { *Variacione } \\
\text { s en la } \\
\text { enseñanza } \\
\text { disciplinar }\end{array}$ & & & & \\
\hline 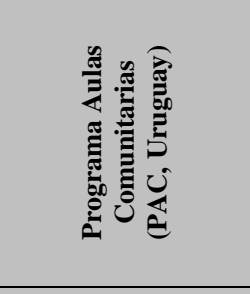 & & & & & 2006 y cont. & $\begin{array}{l}\text { Jóvenes } 12 \text { a } \\
15 \text { fuera del } \\
\text { SE o en riesgo } \\
\text { de abandono }\end{array}$ & $\begin{array}{l}\text { *Reinsertar } \\
\text { en liceo } \\
\text { *Desarroll } \\
\text { ar } \\
\text { innovación } \\
\text { pedagógica }\end{array}$ & $\begin{array}{l}\text { *Cert.ciclo } \\
\text { básico } \\
\text { *Apoyo vida } \\
\text { liceal } \\
\text { *Acompaña } \\
\text { miento } \\
\text { egreso } \\
\text { espacio } \\
\text { puente }\end{array}$ \\
\hline 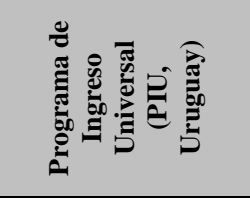 & $\begin{array}{l}2008 \text { y } \\
\text { cont. }\end{array}$ & \begin{tabular}{|l|} 
Escuelas \\
alumnos \\
vulnerabili \\
zados y \\
repitencia \\
(82 liceos) \\
\end{tabular} & $\begin{array}{l}* \text { Mejorar } \\
\text { aprendizaje } \\
\mathrm{s} \\
* \text { Mejorar } \\
\text { promoción }\end{array}$ & $\begin{array}{l}\text { *Tutorías } \\
\text { *Recursos } \\
\text { materiales a } \\
\text { estudiantes } \\
\text { *Atención } \\
\text { psicosocial }\end{array}$ & & & & \\
\hline
\end{tabular}




\begin{tabular}{|c|c|c|c|c|c|c|c|c|}
\hline \multirow{2}{*}{$\begin{array}{l}\text { Tipo } \\
\text { Caso }\end{array}$} & \multicolumn{4}{|c|}{ Sostenimiento de la trayectoria escolar } & \multicolumn{4}{|c|}{ Re-ingreso a la escuela secundaria } \\
\hline & $\begin{array}{c}\text { Período de } \\
\text { implement } \\
\text { ación }\end{array}$ & $\begin{array}{c}\text { Población } \\
\text { objeto }\end{array}$ & $\begin{array}{c}\text { Ejes de la } \\
\text { intervención }\end{array}$ & $\begin{array}{l}\text { Líneas de } \\
\text { acción }\end{array}$ & $\begin{array}{c}\text { Período de } \\
\text { implementación }\end{array}$ & Población objeto & $\begin{array}{c}\text { Ejes de la } \\
\text { intervención }\end{array}$ & $\begin{array}{l}\text { Líneas de } \\
\text { acción }\end{array}$ \\
\hline 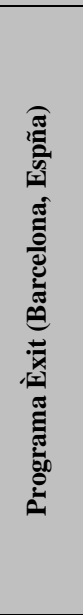 & $\begin{array}{l}2001 \text { y } \\
\text { continúa. }\end{array}$ & $\begin{array}{l}\text { Escuelas } \\
\text { primarias y } \\
\text { secundaria } \\
\text { s } \\
\text { (Institutos) } \\
\text { estudiantes } \\
\text { de } 5 \text { to y } 60 \\
\text { de primaria } \\
\text { y 1ero y } \\
2 \text { do de } \\
\text { secundaria }\end{array}$ & $\begin{array}{l}\text { *Mejorar } \\
\text { el pasaje } \\
\text { entre } \\
\text { primaria y } \\
\text { secundaria. } \\
\text { *Promover } \\
\text { proyectos } \\
\text { de atención } \\
\text { tutorial } \\
\text { individual. } \\
\text { *Vincular } \\
\text { a la familia } \\
\text { y al } \\
\text { entorno en } \\
\text { el } \\
\text { sostenimie } \\
\text { nto de la } \\
\text { trayectoria } \\
\text { escolar. }\end{array}$ & $\begin{array}{l}\text { *Programa } \\
\text { de refuerzo } \\
\text { escolar dos } \\
\text { veces por } \\
\text { semana con } \\
\text { tutores y ex } \\
\text { estudiantes } \\
\text { *Actividade } \\
\text { s } \\
\text { complement } \\
\text { arias para el } \\
\text { desarrollo } \\
\text { de las } \\
\text { competencia } \\
\text { s básicas } \\
\text { (lectura, } \\
\text { radio, teatro, } \\
\text { etc.) }\end{array}$ & & & & \\
\hline 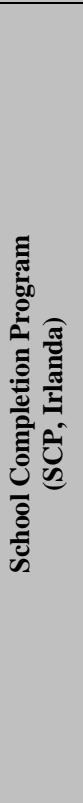 & $\begin{array}{l}2002 \text { y } \\
\text { continúa. }\end{array}$ & $\begin{array}{l}\text { Todos los } \\
\text { estudiantes } \\
\text { calificados } \\
\text { en "riesgo } \\
\text { educativo". }\end{array}$ & $\begin{array}{l}\text { *Retener a } \\
\text { los } \\
\text { estudiantes } \\
\text { hasta los } \\
16 \text { años } \\
\text { (Senior } \\
\text { cycle o } \\
\text { equivalente } \\
\text { ). }\end{array}$ & $\begin{array}{l}\text { *Apoyo } \\
\text { dentro del } \\
\text { Centro: } \\
\text { apoyo extra } \\
\text { en horario } \\
\text { escolar. } \\
\text { *Apoyo } \\
\text { extra clase: } \\
\text { apoyo } \\
\text { adicional } \\
\text { fuera del } \\
\text { horario } \\
\text { escolar. } \\
\text { *Apoyo en } \\
\text { vacaciones: } \\
\text { apoyo a } \\
\text { través de } \\
\text { servicios } \\
\text { juveniles. } \\
\text { *Apoyo } \\
\text { fuera de la } \\
\text { escuela: } \\
\text { para } \\
\text { estudiantes } \\
\text { desertores. }\end{array}$ & & & & \\
\hline
\end{tabular}

Fuente: Elaboración propia en base a documentación sobre los programas seleccionados

\subsection{Análisis de los programas}

Al comienzo de este trabajo se señalaron confluencias y divergencias entre la configuración y expansión de los sistemas educativos latinoamericanos y aquella de los países de modernización temprana. Respecto de la expansión de la escuela secundaria, se indicó que esa heterogeneidad se tradujo en la existencia de sistemas que avanzaron más rápidamente que otros en ese proceso, aunque con fuertes dificultades para la renovación del modelo institucional.

La heterogeneidad de situaciones a atender sumada a la variada historia de las configuraciones educativas locales también se expresa en los programas estudiados. En efecto, dichos programas presentan aspectos comunes y divergentes. Los primeros se vinculan con el problema del acceso y permanencia en la escuela secundaria. Tal como señala Finnegan (2007) los programas se enmarcan en el propósito general de garantizar al menos 12 años de escolarización, de allí el énfasis en el acceso y la finalización del nivel secundario. 
Si se enfocan las políticas y programas destinados a los jóvenes, se observan las siguientes características comunes (en base a Finnegan, 2007 y Acosta, 2013):

- Articulación de la expansión de la escuela secundaria con objetivos de ampliación de derechos sociales.

- Extensión de la escuela secundaria en el marco de la ampliación y cumplimiento del Derecho a la educación.

- Articulación de la escuela secundaria común con la formación para el mundo del trabajo.

- Focalización en grupos poblacionales con tránsito entre la primaria y secundaria interrumpido, en sectores que abandonaron el nivel secundario o que cursan en condiciones de sobreedad y desfasaje etáreo.

- Flexibilización del modelo institucional de la escuela secundaria (en el currículum y en el régimen académico).

- Creación de figuras de apoyo y sostén en la escuela secundaria para acompañar la inserción y el tránsito por la escolaridad.

- Desarrollo y experimentación de formatos de atención educativa alternativos o complementarios (articulables) con el de la educación secundaria escolarizada.

- Contextualización de la oferta en el ámbito local.

- Desarrollo de programas con base en las escuelas y organizaciones locales (generación de capacidad institucional).

Más allá de estos elementos comunes se encuentran ciertas divergencias, muchas de ellas ligadas a la configuración educativa particular. En este sentido, puede proponerse una recategorización de los programas estudiados en cuatro grupos:

- Sostenimiento modalidad extensiva: programas de desarrollo de estrategias para mantener a los estudiantes en su conjunto en la escuela secundaria. Programas: Combatiendo el abandono escolar (Holanda); PMI (Argentina).

- Sostenimiento modalidad segmentada: programas de desarrollo de estrategias de apoyo a grupos vulnerabilizados para mantener a los estudiantes en la escuela secundaria. Programas: PIU (Uruguay), Èxit, (Barcelona), SCP (Irlanda).

- Re ingreso/Adaptación a la escuela secundaria común: programas puente para la reinserción en la escuela secundaria; suponen "adaptación" al dispositivo escolar. Programas: CESAJ (Argentina), PAC (Uruguay).

- Reingreso al sistema educativo: programas de creación de oferta institucional alternativa dentro del sistema de educación formal: no se presentan en este estudio (véase Acosta, 2011a; Acosta, 2014).

Estos cuatro grupos se encuentran atravesados por la distinción, ya señalada por Terigi (2012), entre políticas extensivas y políticas intensivas respecto de la expansión de la escuela secundaria y el cambio o alteración del modelo institucional. Tal como destaca la autora, las políticas extensivas son altamente generalizables, pero no alteran esencialmente el dispositivo escolar: son políticas de perfeccionamiento del dispositivo para sostener la trayectoria de los estudiantes (Acosta, 2013). En este estudio corresponden a este tipo de políticas los programas Combatiendo el abandono escolar de Holanda o el PMI de Argentina.

Las políticas menos extensivas, por su escala acotada, permiten un mayor grado de experimentación e innovación en los cambios sobre el régimen académico, en las modalidades de acompañamiento y seguimiento y en el currículum: son políticas de alteración -en el sentido de flexibilización- del dispositivo para evitar los núcleos duros que están en la 
base del rezago y el abandono. Serían ejemplos de este tipo de política los programas como CESAJ, de la Provincia de Buenos Aires, Argentina y el PAC de Uruguay.

La agenda múltiple que enfrenta la expansión de la enseñanza secundaria hace que sea posible encontrar combinaciones de ambos tipos de políticas. Los programas como el PMI constituyen un ejemplo de política extensiva con baja capacidad de innovación del modelo institucional y con dificultades para su perfeccionamiento. La creación y expansión de los programas como PAC o CESAJ constituyen un ejemplo de política intensiva: de menor escala propone la alteración de algunos rasgos del modelo institucional. Entre unas y otras se encuentran programas fuertemente centrados en el sostenimiento de los estudiantes sin provocar grandes transformaciones en el modelo institucional, tal es el caso del SPC en Irlanda o Exit en Barcelona.

Si se combinan los grupos propuestos con el tipo de política (extensiva o intensiva) y su correlato en los cambios en el modelo institucional (perfeccionamiento o alteración) se obtiene que en un extremo estarían los programas de sostenimiento de modalidad extensiva y en el otro los programas en modalidad alternativa; entre los primeros el dispositivo institucional se mantiene menos alterado, en la medida que se avanza hacia el extremo opuesto se aprecia un grado creciente de alteración.

Las experiencias analizadas revelan distintas formas de acercamiento al problema de la expansión de la escuela secundaria y el modelo institucional, desde el perfeccionamiento del dispositivo escolar en su forma moderna hasta la alteración total (o, al menos, buena parte de ella). A continuación, se presenta una tabla con el conjunto de los programas y las modificaciones específicas a distintos aspectos del modelo institucional: 
Tabla 2. Los programas seleccionados y los cambios en el modelo institucional

\begin{tabular}{|c|c|c|c|c|c|c|c|c|}
\hline $\begin{array}{l}\text { Programa } \\
\text { Dimensión } \\
\text { MI }\end{array}$ & $\begin{array}{l}\text { Apoyo a } \\
\text { establecimi } \\
\text { entos } \\
\text { (Chile) }\end{array}$ & $\begin{array}{l}\text { CESAJ } \\
\text { (Buenos } \\
\text { Aires, } \\
\text { Argentina) }\end{array}$ & $\begin{array}{l}\text { Combatien } \\
\text { do el } \\
\text { abandono } \\
\text { escolar } \\
\text { (Holanda) }\end{array}$ & $\begin{array}{l}\text { PMI } \\
\text { (Argentina } \\
\text { ) }\end{array}$ & $\begin{array}{l}\text { PAC } \\
\text { (Uruguay) }\end{array}$ & $\begin{array}{l}\text { PIU } \\
\text { (Uruguay) }\end{array}$ & $\begin{array}{l}\text { Programa } \\
\text { Exit } \\
\text { (Barcelona, } \\
\text { España) }\end{array}$ & $\begin{array}{l}\text { SCP } \\
\text { (Irlanda) }\end{array}$ \\
\hline $\begin{array}{l}\text { Modalidad } \\
\text { de } \\
\text { acompaña } \\
\text { miento }\end{array}$ & $\begin{array}{l}\text { Asesoría a } \\
\text { los liceos } \\
\text { en área } \\
\text { instituciona } \\
\text { 1, r } \\
\text { pedagógica } \\
\text { y r de } \\
\text { convivencia } \\
\text { en alianza } \\
\text { con } \\
\text { universidad } \\
\text { es }\end{array}$ & $\begin{array}{l}\text { Se incluye } \\
\text { la figura del } \\
\text { tutor. } \\
\text { Participan } \\
\text { agentes e } \\
\text { institucione } \\
\text { s ajenas al } \\
\text { ámbito } \\
\text { escolar. } \\
\text { Hay } \\
\text { profesores } \\
\text { del nivel } \\
\text { secundario, } \\
\text { instructores } \\
\text { de } \\
\text { formación } \\
\text { profesional } \\
\text { y } \\
\text { preceptores. }\end{array}$ & $\begin{array}{l}\text { Número de } \\
\text { educación } \\
\text { personal } \\
\text { para } \\
\text { seguimiento } \\
\text { digital de } \\
\text { control del } \\
\text { absentismo. }\end{array}$ & $\begin{array}{l}\text { Se incluye } \\
\text { la figura del } \\
\text { profesor } \\
\text { tutor. } \\
\text { Implementa } \\
\text { ción de } \\
\text { estrategias } \\
\text { específicas } \\
\text { para } \\
\text { alumnos } \\
\text { con } \\
\text { sobreedad. } \\
\text { Articulació } \\
\mathrm{n} \text { primario } \\
\text { y superior. } \\
\text { Itinerarios } \\
\text { pedagógico } \\
\text { s }\end{array}$ & $\begin{array}{l}\text { Diversas } \\
\text { figuras para } \\
\text { la } \\
\text { enseñanza y } \\
\text { acompañam } \\
\text { iento por } \\
\text { modalidad: } \\
\text { profesores } \\
\text { de liceo, } \\
\text { coordinador } \\
\text { del aula, } \\
\text { trabajador } \\
\text { social, } \\
\text { educador } \\
\text { social y } \\
\text { talleristas }\end{array}$ & $\begin{array}{l}\text { Horas } \\
\text { extracurricu } \\
\text { lares por } \\
\text { docente; } \\
\text { figura del } \\
\text { profesor } \\
\text { referente; } \\
\text { equipos } \\
\text { Multidiscipl } \\
\text { inarios } \\
\text { (Psicólogos, } \\
\text { Asistentes } \\
\text { Sociales y } \\
\text { Educadores } \\
\text { sociales), } \\
\text { con mación } \\
\text { asignaciós } \\
\text { de mara } \\
\text { horas para } \\
\text { estas tareas. }\end{array}$ & $\begin{array}{l}\text { Desarrollo } \\
\text { de } \\
\text { proyectos } \\
\text { de tutoría } \\
\text { por centro } \\
\text { para } \\
\text { seguimiento } \\
\text { de alumnos } \\
\text { en } \\
\text { dificultad, } \\
\text { horas de } \\
\text { refuerzo, } \\
\text { actividades } \\
\text { complemen } \\
\text { tarias para } \\
\text { desarrollo } \\
\text { competenci } \\
\text { as básicas }\end{array}$ & $\begin{array}{l}\text { Todo el } \\
\text { programa } \\
\text { supone un } \\
\text { refuerzo del } \\
\text { acompañam } \\
\text { iento } \\
\text { escolar y } \\
\text { extraescolar } \\
\text {. }\end{array}$ \\
\hline $\begin{array}{l}\text { Funciones } \\
\text { de apoyo al } \\
\text { alumno }\end{array}$ & $\begin{array}{l}\text { Se proveen } \\
\text { materiales } \\
\text { de estudio, } \\
\text { equipos, } \\
\text { textos, } \\
\text { bibliotecas } \\
\text { y horas } \\
\text { docentes }\end{array}$ & 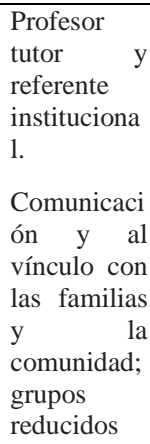 & $\begin{array}{l}\text { Coordinado } \\
\text { res de } \\
\text { cuidados } \\
\text { socioeducat } \\
\text { ivos. } \\
\text { Mentoring } \\
\text { y coaching } \\
\text { para } \\
\text { orientación } \\
\text { laboral. }\end{array}$ & $\begin{array}{l}\text { Se asignan } \\
\text { recursos } \\
\text { para que los } \\
\text { profesores } \\
\text { asuman } \\
\text { tutorías y } \\
\text { horas extra } \\
\text { clase }\end{array}$ & $\begin{array}{l}\text { Grupos } \\
\text { reducidos } \\
\text { para el } \\
\text { seguimiento } \\
\text { en el aula y } \\
\text { re inserción } \\
\text { en la } \\
\text { escuela; } \\
\text { apoyo extra } \\
\text { clase para } \\
\text { egresados } \\
\text { ya en la } \\
\text { escuela }\end{array}$ & $\begin{array}{l}\text { Profesor } \\
\text { referente } \\
\text { instituciona } \\
1, \text { Profesor } \\
\text { tutor; } \\
\text { equipos de } \\
\text { asistencia } \\
\text { social. } \\
\text { Apoyo } \\
\text { recursos } \\
\text { materiales a } \\
\text { alumnos en } \\
\text { riesgo }\end{array}$ & $\begin{array}{l}\text { Creación de } \\
\text { dos figuras } \\
\text { centrales: } \\
\text { tutores en } \\
\text { las escuelas } \\
\text { y amicgrands } \\
\text {, ex } \\
\text { estudiantes, } \\
\text { para las } \\
\text { horas de } \\
\text { refuerzo }\end{array}$ & $\begin{array}{l}\text { Tutores } \\
\text { para apoyo } \\
\text { extra en } \\
\text { horarios } \\
\text { escolar y } \\
\text { extraescolar } \\
\text {, durante las } \\
\text { vacaciones } \\
\text { y para } \\
\text { estudiantes } \\
\text { desertores. }\end{array}$ \\
\hline $\begin{array}{c}\text { Cambios } \\
\text { organizacio } \\
\text { nales }\end{array}$ & $\begin{array}{l}\text { Horas } \\
\text { docentes. } \\
\text { Apoyo a los } \\
\text { estudiantes } \\
\text { en las } \\
\text { universidad } \\
\text { es los días } \\
\text { sábados }\end{array}$ & $\begin{array}{l}\text { Espacio } \\
\text { puente } \\
\text { asociado a } \\
\text { una escuela } \\
\text { secundaria }\end{array}$ & $\begin{array}{l}\text { VM2: } \\
\text { continuar } \\
\text { con la } \\
\text { educación } \\
\text { profesional } \\
\text { en el mismo } \\
\text { centro. }\end{array}$ & No hay & $\begin{array}{l}\text { Espacio } \\
\text { puente para } \\
\text { re inserción } \\
\text { en escuela } \\
\text { secundaria } \\
\text { (liceo) }\end{array}$ & No hay & $\begin{array}{l}\text { Proyectos } \\
\text { de } \\
\text { articulación } \\
\text { entre } \\
\text { escuelas } \\
\text { primarias y } \\
\text { secundarias. } \\
\text { Universidad } \\
\text { es forman } \\
\text { tutores }\end{array}$ & \\
\hline $\begin{array}{c}\text { Cambios } \\
\text { en el } \\
\text { régimen } \\
\text { académico }\end{array}$ & No hay & $\begin{array}{l}\text { Cursada } \\
\text { cuatrimestr } \\
\text { al, } \\
\text { promoción } \\
\text { por materia, } \\
\text { flexibilidad } \\
\text { asistencia }\end{array}$ & & No hay & $\begin{array}{l}\text { Semestraliz } \\
\text { ación, } \\
\text { ajuste carga } \\
\text { horaria por } \\
\text { materia }\end{array}$ & 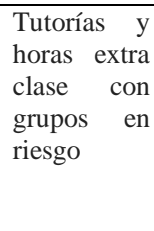 & No hay & \\
\hline
\end{tabular}

Fuente: Elaboración propia en base a documentación sobre los programas seleccionados

Si se comparan los elementos de cambio comunes para el modelo institucional se destacan modificaciones de alcance general y modificaciones de alcance particular, es decir a nivel de la institución escolar: 
Modificaciones generales:

- Designación de profesores por fuera de la hora clase (profesores con asignación de horas institucionales, profesores o monitores para el apoyo extraescolar o recuperación de aprendizajes, profesores tutores (función ya existente);

- Armado de equipos técnicos de gobierno central y/o local de asesoramiento, acompañamiento y trabajo conjunto con los actores en las escuelas; articulación con universidades para formación de recursos en las escuelas

- Revisión general del currículum para diseñar trayectos alternativos, cursadas paralelas y, a la vez, ofrecer conocimientos comunes a todos.

\section{Modificaciones específicas:}

- Flexibilización de disposiciones académicas: horarios de materias, formas de cursada, oferta curricular, recuperación de materias en paralelo al cursado de año o nivel;

- Oferta de cursos y programas de interés para los alumnos; ampliación de la oferta formativa (escuelas de verano, actividades complementarias)

- Libre disposición de fondos para el desarrollo de programas extra destinados a la retención de alumnos en riesgo;

- Localización en: sostenimiento del "ritmo" educativo (evitar que el alumno se "desenganche"); apuntalamiento de momentos clave de la trayectoria educativa, especialmente la transición ente la primaria y la secundaria y entre ésta y estudios o trabajos posteriores (evitar que la escuela pierda "relevancia" en un proyecto de continuidad).

La dimensión sistémica permite comprender que el problema de falta de eficiencia en el sostenimiento de las trayectorias educativas no se ancla en un solo nivel educativo. Se observa a nivel internacional una tendencia cada vez más robusta a considerar los desacoplamientos en las trayectorias escolares ya no como un problema individual de los sujetos, sino como un problema que debe ser atendido sistémicamente (Terigi, 2012). Las iniciativas pueden valorarse en sus aportes a la detección de dificultades en las trayectorias y en sus acciones para intervenir frente a ellas. En este sentido, el desarrollo de dispositivos que permitan alertas tempranas vinculadas con el ausentismo reiterado, la no promoción y el abandono constituyen herramientas contemporáneas en uso para favorecer el seguimiento de las trayectorias. Así lo muestra el programa Combatiendo el Abandono escolar de Holanda. (Acosta y Terigi, 2015).

Asimismo, se evidencian estrategias para que los estudiantes no se vuelvan invisibles. Los tutores y acompañantes para refuerzo escolar (los amicgrands en el caso de Éxit) y coordinadores socioeducativos en varios programas son ejemplo de las nuevas formas de atención a este problema.

Los programas analizados muestran distintas formas y grados de alcance al momento de implementar cambios en el modelo institucional de la escuela secundaria. Tal y como se desarrolla en el siguiente apartado, los límites frente a dichos alcances, se encuentran asociados a las continuidades históricas en la forma de organización de la escuela secundaria. 


\section{Los programas entre los cambios y las continuidades: conclusiones sobre las formas actuales de expansión de la escuela secundaria}

A lo largo de este trabajo se presentaron algunos desafíos para la extensión de la enseñanza secundaria. Para abordar el problema del cambio en la escuela secundaria se propuso como argumento principal la corroboración de la existencia de dificultades comunes en los sistemas educativos de Occidente en relación con el par expansión-modelo institucional de la escuela secundaria. Esto significa que a medida que el completamiento de niveles básicos de escolarización fue presionando sobre la expansión de la educación secundaria, ciertas características de la organización tradicional de ésta generaron dificultades para retener a todos los estudiantes y asegurar la certificación de los estudios. Históricamente, las políticas de extensión de la escolarización secundaria han encontrado problemas de ajuste entre los modelos institucionales y los nuevos sectores sociales que acceden a ese nivel.

Los programas analizados no escapan a este riesgo que corre toda política destinada a la extensión de la escuela secundaria. El problema exige el desarrollo de modalidades alternativas al momento de considerar la extensión de la escolarización. Después de todo, los sistemas educativos modernos son inventos con una historia reciente cuyo origen se sitúa en Europa Occidental. La clave para el éxito de dichos sistemas fue la capacidad de diseñar un dispositivo de alfabetización masiva como la escuela. Pero la escuela y la escolarización suponen y generan condiciones al mismo tiempo. Cuando esas condiciones no son posibles (o deseables) muchos países desarrollan alternativas para la distribución de saberes.

El problema es que esas alternativas también suponen y generan condiciones: suponen recursos físicos y humanos y generan la demanda por sostener el carácter innovador respecto de la oferta escolar tradicional. Si estas condiciones no se satisfacen pueden perder su sentido y agravar la fragmentación del sistema de distribución de saberes existente, la que se apoya sobre la falencia en la dinámica de sistematización del sistema educativo (articulación del sistema) y potencia la segmentación (diferenciación escolar sobre la base de la desigualdad social).

Las modalidades flexibles como las analizadas en este estudio, podrían estar contribuyendo a aumentar la fragmentación y segmentación del sistema educativo, riesgo que corre toda política intensiva destinada al nivel medio. Por un lado, se advierte el riesgo de una segmentación socioeconómica en las políticas que parten de la identificación de alumnos en situación de vulnerabilidad social y educativa y proponen ofertas para esos grupos. Crear "escuelas para pobres" puede transformarse en un efecto no deseado de la intervención. Por otro lado, se observa también un riesgo de segmentación pedagógica en la medida en que se proponen ofertas curriculares destinadas a alumnos con dificultades sociales y académicas.

En consonancia con el riesgo anterior, las políticas que parten de la focalización corren el riesgo de producir nuevas etiquetas que pueden transformarse en estigmas. Aquí el efecto ya no sería sobre la escuela o el sistema como en el caso anterior, si no que afectaría en forma directa al alumno que recibe la etiqueta. Toda oferta que diferencia grupos (por escuela o dentro de la escuela) también produce estigmas y las escuelas secundarias, en particular, tienen una larga historia en este tipo de dinámica de manera que 
el riesgo en los programas analizados es la articulación con esta dinámica propia de la escuela secundaria moderna. De esta manera, políticas de innovación que apuntan a evitar los problemas del sistema escolar tradicional pueden en realidad contribuir a profundizarlos.

En las experiencias analizadas se encuentran arreglos pedagógicos y didácticos flexibles; aunque las iniciativas pueden ordenarse entre dos extremos, desde perfeccionar el dispositivo existente hasta su alteración, existe un grado de conciencia importante acerca de la necesidad de revisarlo (Acosta, 2013). Las articulaciones de este tipo tendrían la potencialidad de presentar muchas opciones de flexibilidad en cuanto a instituciones y condiciones para aprender (tiempos, espacios, recursos, figuras docentes, etc.). El riesgo de la fragmentación educativa y la segmentación escolar igual está allí.

Estudios previos (Acosta, 2011a; Acosta, 2013) destacan ciertos elementos al momento de considerar políticas de cambio para la enseñanza secundaria:

- Previsión y provisión de recursos humanos y financieros

- Continuidad de políticas previas y contemporáneas

- Articulación con políticas sociales y laborales

- Mirada sobre el Modelo institucional

- Búsqueda de legitimación institucional

- Focalización sobre trayectorias continuas

Este trabajo agrega tres orientaciones generales al momento de considerar el desafío de la extensión de la escuela secundaria. La primera orientación refiere a la adopción de una mirada sistémica, sobre todo al momento de considerar la cuestión de las trayectorias escolares. El fortalecimiento de programas de apoyo a la escolarización (becas) desde la primaria y durante la escuela secundaria, así como el desarrollo de dispositivos de seguimiento en el pasaje de un nivel educativo a otro y sobre la trayectoria en la escuela secundaria parecen puntos clave.

La segunda refiere a la necesidad de revisar las formas históricas y contemporáneas de la oferta de enseñanza. Más jóvenes en la escuela secundaria siempre es un dato positivo. Pero también implica un desafío: significa revisar las prácticas existentes porque muchas veces son las condiciones de la escolarización (tanto en su régimen académico como en las formas de enseñanza) las que expulsan a los nuevos estudiantes. Al respecto, la experiencia internacional en la expansión de la escuela secundaria revela modificaciones importantes en cuatro direcciones:

- Modalidades de acompañamiento (actores destinados al seguimiento de los jóvenes en situación de vulnerabilidad respecto a la trayectoria escolar);

- Efectos de alteración en el modelo institucional en función del desarrollo de modalidades de acompañamiento;

- Cambios sobre el régimen académico a partir de la incorporación de modalidades que trabajan sobre las trayectorias;

- Aprovechamiento de medios disponibles en la actualidad -redes sociales- por parte del programa bajo implementación para trabajar sobre las trayectorias de los jóvenes. 
La tercera orientación refiere a considerar una perspectiva de internacionalización de la escolarización al momento de analizar alternativas. Se considera que toda propuesta puede ser enriquecida a partir del análisis de otras experiencias las que deben ser contextualizadas en función de la historia, las tradiciones y las características de los sistemas educativos y sus instituciones en el ámbito local; esto es la relación entre tendencias de internacionalización y lógicas de apropiación en la expansión de la escolarización. La relación entre diferentes escalas como la global y la regional remite al problema del contexto: tratar de comprender la confluencia de prácticas y objetos que entran en contacto en procesos de constante mutación (Sobe y Kowalczyk, 2012). En este sentido, no se trata de construir listados o agendas de "buenas prácticas" si no de considerar experiencias de cambio y sus posibles alteraciones en la circulación entre un escenario y otro.

\section{Referencias bibliográficas}

Acosta, F. y D. Pinkasz (2007). La escuela media y las necesidades de la masificación. Argentina: Ministerio de Educación. Mimeo.

Acosta, F. (2011a). La educación secundaria en foco: análisis de políticas de inclusión en Argentina, Canadá, Chile y España. Buenos Aires: IIPE UNESCO Buenos Aires.

Acosta, F. (2011b). Escuela secundaria y sistemas educativos modernos: análisis histórico comparado de la dinámica de configuración y expansión en países centrales y en la Argentina. HISTEDBR, 42, 3-13, doi: 10.20396/rho.v11i42.8639863

Acosta, F. (2012). La escuela secundaria argentina en perspectiva histórica y comparada: modelos institucionales y desgranamiento durante el siglo XX. Cadernos de História da Educaçao, 11 (1), 131-144. Recuperado de: http://www.seer.ufu.br/index.php/che/article/view/17534/9633

Acosta, F. (2013). Trabajo analítico (multi país) sobre experiencias de cambio en la escuela secundaria con foco en políticas destinadas a la reinserción y permanencia de los jóvenes en la escuela en América Latina (Cono Sur). Informe de consultoría para OEI/EUROsociAL.

Acosta, F. (2014). Entre procesos globales y usos locales: análisis de categorías recientes de la historia de la educación para el estudio de la escuela secundaria en la Argentina. Revista Tiempo, Espacios, Educación, 1 (2), 23-37, doi: 10.14516/ete

Acosta, F. y Terigi, F. (2015). Experiencias de cambio en la escuela secundaria con foco en las políticas destinadas a la reinserción y permanencia de los jóvenes en la escuela en América Latina y en Europa. Madrid: OEI/EUROsoCIAL.

European Commission/ Directorate-General for Education and Culture (2012). Conference report: reducing early school leaving: efficient and effective policies in Europe. Recuperado 
http://ec.europa.eu/dgs/education_culture/repository/education/events/2012/docum ents/esl-report_en.pdf

European Commission/EACEA/Eurydice/Cedefop (2014). Tackling Early Leaving from Education and Training in Europe: Strategies, Policies and Measures. Eurydice and Cedefop Report. Luxembourg: Publications Office of the European Union.

Campbell, C. y Sherrington, G. (2006). The Comprehensive Public High School. Historical perspectives. New York: Palgrave Macmillan.

Caruso, M. y Tenorh, H. (2011). Introducción: conceptualizar e historizar la internacionalización y la globalización en el campo educativo. En M. Caruso, M. y H. Tenorh (Eds.), Internacionalización. Políticas educativas y reflexión pedagógica en un medio global (pp. 13-35). Buenos Aires: Granica.

Fernández Enguita, M. (1985). Integrar o segregar. La enseñanza secundaria en los países industrializados. Barcelona: Laia.

Finnegan, F. (2007). Tendencias recientes en políticas y programas que apuntan a la finalización de la escolaridad secundaria en América Latina. En Tendencias en foco, n⿳3 3 . Red Etis. Recuperado de: http://www.redetis.iipe.unesco.org.

Lyche, C. (2010). Taking on the Completion Challenge: A Literature Review on Policies to Prevent Dropout and Early School Leaving. OECD Education Working Papers, No. 53. OECD Publishing. Recuperado de: http://www.oecdilibrary.org/education/taking-on-the-completion-challenge_5km4m2t59cmr-en

Mueller, F. (1992). El proceso de sistematización: el caso de la educación secundaria en Alemania. En D. Mueller, F., Ringer y B. Simon (Comps.). El desarrollo del sistema educativo moderno. Cambio estructural y reproducción social 1870-1920 (pp. 37-86). Madrid: Ministerio de Trabajo y Seguridad.

Ringer, F. (1992). La segmentación en los sistemas educativos modernos. En D. Mueller, F., Ringer y B. Simon (Comps.). El desarrollo del sistema educativo moderno. Cambio estructural y reproducción social 1870-1920 (pp.161-194). Madrid: Ministerio de Trabajo y Seguridad.

Schriewer, J. (2002). Educación comparada: un gran programa ante nuevos desafíos. En J. Schriewer (Comp.) Formación del discurso en la educación comparada, (pp. 1340). Barcelona: Pomares-Corredor.

Schriewer, J. (2010). Comparación y explicación entre causalidad y complejidad. En J. Schriewer y H. Kaelbe (Comp.) La comparación en las ciencias sociales e históricas. Un debate interdisciplinar (pp. 17-62). Barcelona: Octaedro/ICE-UB.

SITEAL (2010). Metas educativas 2021: Desafíos y oportunidades, 2010. Recuperado de: http://www.siteal.iipe-oei.org/informe/227/informe-2008 
Sobe, N.W. y Kowalczyk, J. (2012). The problem of context in comparative educational research. ECPS journal, 6, 51-74, doi: 10.7358/ecps-2012-006-sobe

Steedman, H. (1992). Instituciones determinantes: las "endowed grammar schools" y la sistematización de la educación secundaria inglesa. En D. Mueller, F., Ringer y B. Simon (Comps.). El desarrollo del sistema educativo moderno. Cambio estructural y reproducción social 1870-1920 (pp. 161-195). Madrid: Ministerio de Trabajo y Seguridad.

Thröhler, D. y Lenz, Th. (2015). Trayectoria del desarrollo de la escuela moderna. Entre lo nacional y lo global: Introducción. En D. Tröhler y Th. Lenz (Comps.) Trayectorias del desarrollo de los sistemas educativos modernos. Entre lo nacional y lo global (pp.11-19). Barcelona: Octaedro.

Terigi, F. (2012). La escolarización secundaria de adolescentes y jóvenes en América Latina. Aportes para el análisis de la situación y la deliberación de políticas. Madrid: Eurosocial/OEI. .

Viñao, A. (2002). Sistemas educativos, culturas escolares y reformas. Madrid: Morata.

Weiler, H. (1998). ¿Por qué fracasan las reformas: Política educativa en Francia y República Federal de Alemania?. Revista Estudios del Currículo, 1(2), 54-76.

Wiborg, S. (2009). Education and social integration. Comprehensive Schooling in Europe. USA: Palgrave MacMillan

Wilson S.J, Tanner-Smith E., Lipsey, M.W, Steinka-Fry, K y Morrison, J. (2011). Dropout prevention and intervention programs: Effects on school completion and dropout among school aged children and youth. Campbell Systematic Reviews. Doi: 10.4073/csr.2011.8 